\title{
High Balanced Biorthogonal Multiwavelets with Symmetry
}

\author{
Youfa Li, ${ }^{1}$ Shouzhi Yang, ${ }^{2}$ Yanfeng Shen, ${ }^{3}$ and Gengrong Zhang ${ }^{1}$ \\ ${ }^{1}$ College of Mathematics and Information Sciences, Guangxi University, Nanning 530004, China \\ ${ }^{2}$ Department of Mathematics, Shantou University, Shantou 515063, China \\ ${ }^{3}$ Department of Mathematics, Dezhou University, Dezhou 253023, China
}

Correspondence should be addressed to Yanfeng Shen; shyf8584@163.com

Received 10 June 2014; Accepted 28 August 2014; Published 10 November 2014

Academic Editor: Thanh Tran

Copyright (c) 2014 Youfa Li et al. This is an open access article distributed under the Creative Commons Attribution License, which permits unrestricted use, distribution, and reproduction in any medium, provided the original work is properly cited.

Balanced multiwavelet transform can process the vector-valued data sparsely while preserving a polynomial signal. Yang et al. (2006) constructed balanced multiwavelets from the existing nonbalanced ones. It will be proved, however, in this paper that if the nonbalanced multiwavelets have antisymmetric component, it is impossible for the balanced multiwavelets by the method mentioned above to have symmetry. In this paper, we give an algorithm for constructing a pair of biorthogonal symmetric refinable function vectors from any orthogonal refinable function vector, which has symmetric and antisymmetric components. Then, a general scheme is given for high balanced biorthogonal multiwavelets with symmetry from the constructed pair of biorthogonal refinable function vectors. Moreover, we discuss the approximation orders of the biorthogonal symmetric refinable function vectors. An example is given to illustrate our results.

\section{Introduction}

Multiwavelets have been studied extensively in the literature; for example, to mention only a few here, see [1-7] and the references therein. Implementing multiwavelet transform, one will face a serious problem related to the discrepancy of their approximation properties between the function setting and the discrete vector data setting [1, 8-10]. As such, a multiwavelet must be prefiltered or balanced in advance $[1,2$, $8-10]$. Balanced multiwavelet transform can sparsely process the vector-valued data efficiently. Moreover, they can preserve the polynomial structure of a signal. As such, constructing balanced multiwavelets is of interest for researchers of wavelet analysis. In this paper, we will construct pairs of biorthogonal symmetric multiwavelets with high balanced orders from any orthogonal refinable function vector, which simultaneously has symmetric and antisymmetric components. Before introducing our motivation, let us give some conceptions and notations.

Let $\mathbb{N}_{0}$ denote the set of nonnegative integers while $D^{j} f(x)$ denotes the $j$ th derivative of $f(x), j \in \mathbb{N}_{0}$. Suppose that a compactly supported $d$-refinable function vector $\phi:=$ $\left(\phi_{1}, \ldots, \phi_{r}\right)^{T}: \mathbb{R} \mapsto \mathbb{C}^{r \times 1}$ satisfies the refinement equation

$$
\phi=d \sum_{k \in \mathbb{Z}} a_{k} \phi(d \cdot-k)
$$

with the integer $d(\geq 2)$ being regarded to as a dilation factor. We get from (1) that $\widehat{\phi}(d \xi)=\widehat{a}(\xi) \widehat{\phi}(\xi)$, with $\widehat{a}(\xi)=\sum_{k \in \mathbb{Z}} a_{k} e^{-i k \xi}$ being called the mask symbol of $\phi$ and Fourier transform $\hat{g}$ of $g \in L_{1}(\mathbb{R})$ being defined to be $\widehat{g}(\xi)=\int_{\mathbb{R}} g(x) e^{-i x \xi} d x$, which can be naturally extended to square integrable functions and temper distributions. We say that $\widehat{a}(\xi)$ has $m$ sum rules if there exists a $1 \times r$ matrix $\widehat{Y}(\xi):=\left(\widehat{Y}_{1}(\xi), \widehat{Y}_{2}(\xi), \ldots, \widehat{Y}_{r}(\xi)\right)$ of $2 \pi$-periodic trigonometric polynomials with $\widehat{Y}(0) \neq 0$ such that

$$
\begin{aligned}
& \widehat{Y}(d \xi) \widehat{a}(\xi+2 \pi \gamma)=\delta_{\gamma} \widehat{Y}(\xi)+O\left(|\xi|^{m+1}\right), \\
& \gamma \in\left\{0, \frac{1}{d}, \ldots, \frac{(d-1)}{d}\right\}, \quad m \in \mathbb{N}_{0} .
\end{aligned}
$$


We say that $\phi$ has $m$ approximation orders if there exists a sequence $\left\{\left(C_{k, j}^{1}, C_{k, j}^{2}, \ldots, C_{k, j}^{r}\right)\right\}_{k \in \mathbb{Z}}$ of $1 \times r$ vectors such that, for any polynomial $x^{j}, j=0, \ldots, m-1$, it can be represented as

$$
x^{j}=\sum_{k \in \mathbb{Z}}\left(C_{k, j}^{1}, C_{k, j}^{2}, \ldots, C_{k, j}^{r}\right) \phi(x-k) .
$$

It is well known that $\phi$ has $m$ approximation orders if and only if its mask symbol $\widehat{a}(\xi)$ has $m$ sum rules.

Next, let us introduce the relationship between the mask symbol of a refinable function vector and its symmetry. Suppose that $\phi=\left(\phi_{1}, \ldots, \phi_{r}\right)^{T} \in\left(L_{2}(\mathbb{R})\right)^{r}$ in (1) satisfies

$$
\phi_{j}(x)=\epsilon_{j} \phi_{j}\left(t_{j}-x\right), \quad \epsilon_{j} \in\{1,-1\}, t_{j} \in \mathbb{R} .
$$

Then, it is not difficult to check that

$$
\widehat{a}(\xi)=\widehat{S}(d \xi)^{-1} \overline{\widehat{a}(\xi)} \widehat{S}(\xi)
$$

with $\widehat{S}(\xi)=\operatorname{diag}\left(\epsilon_{1} e^{i t_{1} \xi}, \ldots, \epsilon_{r} e^{i t_{r} \xi}\right), j=1, \ldots, r$. In the words of element, (5) is equivalent to

$$
\widehat{a_{j, k}}(\xi)=\epsilon_{j} \epsilon_{k} e^{-i d\left(t_{j}-t_{k} / d\right) \xi} \widehat{\widehat{a_{j, k}}(\xi)}
$$

where $\widehat{a_{j, k}}(\xi)$ is the $(j, k)$-element of $\widehat{a}(\xi)$. Suppose $\widehat{U}(\xi)$ is an $r \times r$ matrix of $2 \pi$-periodic trigonometric polynomials, which satisfies

$$
\widehat{U}(\xi)=\widehat{\dot{S}}(\xi)^{-1} \overline{\widehat{U}(\xi)} \widehat{S}(\xi)
$$

with $\hat{\dot{S}}(\xi)=\operatorname{diag}\left(\dot{\epsilon}_{1} e^{i \dot{t}_{1} \xi}, \ldots, \dot{\epsilon}_{r} e^{i \dot{t}_{r} \xi}\right), \dot{\epsilon}_{j} \in\{1,-1\}, j=1, \ldots, r$, and then it is not difficult to check that

$$
\widehat{\dot{a}}(\xi)=\widehat{\dot{S}}(d \xi)^{-1} \overline{\hat{\dot{a}}(\xi)} \widehat{\dot{S}}(\xi),
$$

where $\widehat{\dot{a}}(\xi):=\widehat{U}(d \xi) \widehat{a}(\xi) \widehat{U}(\xi)^{-1}$. We will use (7) in the proof of Theorem 3 .

Let $\phi=\left(\phi_{1}, \ldots, \phi_{r}\right)^{T}$ and $\tilde{\phi}=\left(\widetilde{\phi}_{1}, \ldots, \widetilde{\phi}_{r}\right)^{T}$ be two $d$ refinable function vectors in $\left(L_{2}(\mathbb{R})\right)^{r}$. Their mask symbols are $\widehat{a}(\xi)$ and $\widehat{\tilde{a}}(\xi)$, respectively. They are a pair of biorthogonal refinable function vectors, that is,

$$
\left\langle\phi_{\ell}(x-k), \tilde{\phi}_{\ell^{\prime}}(x)\right\rangle=\delta_{k} \delta_{\ell-\ell^{\prime}},
$$

if and only if

$$
\sum_{\ell=0}^{d-1} \widehat{a}\left(\xi+\frac{2 \pi \ell}{d}\right) \hat{\widetilde{a}}\left(\xi+\frac{2 \pi \ell}{d}\right)^{*}=I_{r},
$$

where $*$ denotes the transpose conjugate. When $\phi=\tilde{\phi}$, we call $\phi$ an orthogonal $d$-refinable function vector.

Let $\phi=\left(\phi_{1}, \ldots, \phi_{r}\right)^{T}$ be an orthogonal $d$-refinable function vector. We say that it has $\kappa+1$ balanced orders if

$$
\begin{aligned}
& \int_{\mathbb{R}} \phi_{1}(x) x^{j} d x=\int_{\mathbb{R}} \phi_{2}(x)\left(x-\frac{1}{r}\right)^{j} d x \\
&=\cdots=\int_{\mathbb{R}} \phi_{r}(x)\left(x-\frac{r-1}{r}\right)^{j} d x \\
& \qquad j=0, \ldots, \kappa ; \quad \kappa \in \mathbb{N}_{0} .
\end{aligned}
$$

There are a few equivalent definitions of balanced multiwavelets in the literature; see [11]. In $[10,11]$, the conception of balanced multiwavelets is extended to the case of biorthogonal multiwavelets. Next, we will introduce our motivation. The following remark is necessary.

Remark 1 (a balanced refinable function vector does not has antisymmetric component). Suppose that $\phi$ satisfies (2) and has $\kappa+1$ balanced orders. By (11), we get that

$$
\int_{\mathbb{R}} \phi_{1}(x) d x=\int_{\mathbb{R}} \phi_{2}(x) d x=\cdots=\int_{\mathbb{R}} \phi_{r}(x) d x .
$$

$\widehat{a}(0)$ satisfies Condition $E$; that is, 1 is the simple eigenvalue of $\widehat{a}(0)$ while the other eigenvalues are strictly smaller than 1 in modulus. It follows from (2) that $\widehat{Y}(0) \widehat{\phi}(0) \neq 0$, which leads to the fact that $\int_{\mathbb{R}} \phi_{\ell}(x) d x \neq 0, \ell=1, \ldots, r$, and consequently $\phi$ has no antisymmetric component.

In [8], Yang and Peng constructed balanced multiwavelets via PTST method. Before introducing our motivation, let us briefly introduce the main idea of PTST. Let $\phi$ be an orthogonal $d$-refinable function vector. Construct $\phi_{\natural}=$ $\left(\phi_{\natural 1}, \phi_{\natural 2}, \ldots, \phi_{\natural r}\right)^{T}$ via $\widehat{\phi}_{\natural}(\xi)=\widehat{U}(\xi) \widehat{\phi}(\xi)$ with $\widehat{U}(\xi)$ being a paraunitary matrix; that is, $\widehat{U}(\xi) \widehat{U}(\xi)^{*}=I_{r}$. It is not difficult to check that $\phi_{\natural}$ is still orthogonal. Some conditions are imposed on $\widehat{U}(\xi)$ such that $\phi_{\natural}$ has desired balanced orders [8, Theorem 2, Theorem 4]. However, the balanced $\phi_{\natural}$ can not preserve the symmetry of $\phi$ if it has antisymmetric component. Next, we will explain the reason, which is the motivation of this paper. Without losing generality, assume that

$$
\begin{aligned}
& \phi_{1}(x)=\phi_{1}\left(t_{1}-x\right), \ldots, \phi_{s}(x)=\phi_{s}\left(t_{s}-x\right), \\
& \phi_{j}(x)=-\phi_{j}\left(t_{j}-x\right),
\end{aligned}
$$

$j=s+1, \ldots, r, s>0$. Suppose that there exists a paraunitary matrix $\widehat{U}(\xi):=\left(\widehat{U}_{i, j}(\xi)\right)_{i, j}$ such that $\phi_{\natural}$ is balanced and all the components of $\phi_{\natural}$ have symmetry. By Remark 1 , all the components of $\phi_{\natural}$ are symmetric. Then, it is easy to see that $\widehat{U}_{s+1, \ell}(\xi), \ell=1, \ldots, r$, are all antisymmetric polynomials, which contradicts the fact that $\widehat{U}(0)$ is invertible. In conclusion, there does not exist invertible matrix $\widehat{U}(\xi)$, such that $\phi_{\natural}$ is simultaneously balanced and symmetric if $\phi$ has antisymmetric component.

Since many famous refinable function vectors satisfy (13), for example, CL multiwavelets [12], we naturally face the following problem.

How can we construct symmetric multiwavelets with high balanced orders from such ones that have antisymmetric component?

In this paper, we are interested in the case of (13) with $s=$ 1. That is,

$$
\begin{aligned}
& \phi_{1}(x)=\phi_{1}\left(t_{1}-x\right), \\
& \phi_{2}(x)=-\phi_{2}\left(t_{2}-x\right), \ldots, \phi_{r}(x)=-\phi_{r}\left(t_{r}-x\right) .
\end{aligned}
$$

In fact, many classical multiwavelets satisfy (14). Readers are referred to [13] for many examples. 
We will need the following results related to the canonical form of mask symbols of refinable function vectors.

Lemma 2 (see [2]). Let $\phi:=\left(\phi_{1}, \ldots, \phi_{r}\right)^{T}$ be a compactly supported $d$-refinable function vectors satisfying (1), (2), and (13). Then, for any $n \in \mathbb{N}$, there exists a strongly invertible matrix $\widehat{U}(\xi)$; that is, $\widehat{U}(\xi)$ and $\widehat{U}(\xi)^{-1}$ are both matrices of $2 \pi$ periodic trigonometric polynomials, such that the mask symbol $\widehat{a^{u}}(\xi)=\widehat{U}(d \xi) \widehat{a}(\xi) \widehat{U}(\xi)^{-1}$ takes the form

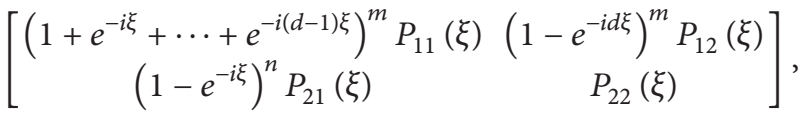

$$
\begin{aligned}
& P_{11}(0)=d^{-m} \text {, }
\end{aligned}
$$

where $P_{11}(\xi), P_{12}(\xi), P_{21}(\xi)$, and $P_{22}(\xi)$ are some $1 \times 1,1 \times$ $(r-1),(r-1) \times 1$, and $(r-1) \times(r-1)$ matrices of $2 \pi$-periodic trigonometric polynomials, respectively. Moreover, $\phi^{u}:=\left(\phi_{1}^{u}, \phi_{2}^{u}, \ldots, \phi_{r}^{u}\right)^{T}$ constructed via

$$
\widehat{\phi^{u}}(\xi)=\widehat{U}(\xi) \widehat{\phi}(\xi)
$$

also satisfies (13) with $\phi_{j}(x)$ being replaced by $\phi_{j}^{u}(x), j=$ $1, \ldots, r$, and $D^{k} \widehat{\phi_{\ell}^{u}}(0)=0, \ell=2, \ldots, r, k=0, \ldots, n-1$.

Note. (I) The algorithm for constructing $\widehat{U}(\xi)$ in Lemma 2 can be seen in the proof of [2, Theorem 2.5]. (II) There exist a number of orthogonal multiwavelets in the literature, whose mask symbols take the form of $(15)$; just see $[12,13]$ for some examples.

\section{Main Results}

Theorem 3. Let $\phi:=\left(\phi_{1}, \ldots, \phi_{r}\right)^{T}$ be an orthogonal compactly supported d-refinable function vector satisfying (1), (2), and (14). Then, for any positive integer $n$, we can construct an $r \times r$ matrix $\widehat{V}(\xi)$ of $2 \pi$-periodic trigonometric polynomials such that the compactly supported d-refinable $\phi_{n}$, which is defined via

$$
\begin{gathered}
\widehat{\phi_{\natural}}(d \xi)=\widehat{a_{\natural}}(\xi) \widehat{\phi_{\natural}}(\xi), \\
\widehat{a_{\natural}}(\xi)=\frac{1}{d} \widehat{V}(d \xi) \widehat{a}(\xi) \widehat{V}(\xi)^{-1},
\end{gathered}
$$

is symmetric and has $n$ balanced orders. Moreover, the $d$ refinable function vector $\widetilde{\phi}_{\natural}$, which is defined via

$$
\begin{aligned}
\widehat{\widetilde{\phi}}_{\natural}(d \xi) & =\widehat{\widetilde{a}}_{\natural}(\xi) \widehat{\widetilde{\phi}}_{\natural}(\xi), \\
\widehat{\widetilde{a}_{\natural}}(\xi) & =d \widehat{V}(d \xi)^{-*} \widehat{a}(\xi) \widehat{V}(\xi)^{*},
\end{aligned}
$$

is also symmetric and a dual refinable function vector of $\phi_{\natural}$. On the other hand, $\phi_{\natural}$ and $\widetilde{\phi_{\natural}}$ have $m+1$ and $m-1$ approximation orders, respectively.

Theorem 4. Let $\phi=\left(\phi_{1}, \ldots, \phi_{r}\right)^{T}$ be as in Theorem 3 . Moreover, its mask symbol $\widehat{a}(\xi)$ takes the form of (15) with $m \neq 0$. Then, $\widetilde{\phi}_{\natural}$ defined by (18) is compactly supported.

\section{Proof of Main Result and Algorithm for High Balanced Biorthogonal Multiwavelets}

Proof of Theorem 3. $\widehat{a}(\xi)$ has $m$ sum rules. Of course, it has 1 sum rule. According to Lemma 2, there exists a strongly invertible matrix $\widehat{U}(\xi)$, such that the mask symbol $\widehat{a^{u}}(\xi)=$ $\widehat{U}(d \xi) \widehat{a}(\xi) \widehat{U}(\xi)^{-1}$ has the property of (15); that is,

$$
\begin{gathered}
\widehat{a^{u}}(\xi) \\
=\left[\begin{array}{cc}
\left(1+e^{-i \xi}+\cdots+e^{-i(d-1) \xi}\right) P_{11}^{1}(\xi) & \left(1-e^{-i d \xi}\right) P_{12}^{1}(\xi) \\
\left(1-e^{-i \xi}\right) P_{21}^{1}(\xi) & P_{22}^{1}(\xi)
\end{array}\right], \\
P_{11}^{1}(0)=d^{-1},
\end{gathered}
$$

where $P_{11}^{1}(\xi), P_{12}^{1}(\xi), P_{21}^{1}(\xi)$, and $P_{22}^{1}(\xi)$ are some $1 \times 1,1 \times$ $(r-1),(r-1) \times 1$, and $(r-1) \times(r-1)$ matrices of $2 \pi$-periodic trigonometric polynomials, respectively. Define $\phi^{u}:=\left(\phi_{1}^{u}, \ldots, \phi_{r}^{u}\right)^{T}$ via

$$
\widehat{\phi^{u}}(\xi)=\widehat{U}(\xi) \widehat{\phi}(\xi)
$$

Then, $\phi^{u}=\left(\phi_{1}^{u}, \ldots, \phi_{r}^{u}\right)^{T}$ satisfies (13). Define $\widehat{H}(\xi):=$ $\left[\begin{array}{cc}\left(1-e^{-i \xi}\right) & O \\ O & I_{r-1}\end{array}\right]$ and $\widehat{\dot{a}}(\xi):=(1 / d) \widehat{H}(d \xi) \widehat{a^{u}}(\xi) \widehat{H}(\xi)^{-1}$. Then, it is not difficult to check that

$\widehat{\dot{a}}(\xi)$

$$
\begin{gathered}
=\frac{1}{d} \widehat{H}(d \xi) \widehat{U}(d \xi) \widehat{a}(\xi) \widehat{U}(\xi)^{-1}(\xi) \widehat{H}(\xi)^{-1} \\
=\left[\begin{array}{cc}
\frac{1}{d}\left(1+e^{-i \xi}+\cdots+e^{-i(d-1) \xi}\right)^{2} P_{11}^{1}(\xi) & \frac{1}{d}\left(1-e^{-i d \xi}\right)^{2} P_{12}^{1}(\xi) \\
\frac{1}{d} P_{21}^{1}(\xi) & \frac{1}{d} P_{22}^{1}(\xi)
\end{array}\right], \\
P_{11}^{1}(0)=d^{-1} .
\end{gathered}
$$

By (21) and (6), we have

$$
\begin{aligned}
& \widehat{\dot{a}_{1,1}}(\xi)=e^{-i d\left((d-1) / d+t_{1} / d\right) \xi} \overline{\widehat{\dot{a}_{1,1}}(\xi)}, \\
& \widehat{a_{1, k}}(\xi)=\epsilon_{1}\left(-\epsilon_{k}\right) e^{-i d\left(t_{1}+1-t_{k} / d\right) \xi} \overline{\widehat{a_{1, k}}(\xi)} \\
& \widehat{a_{j, 1}}(\xi)=-\epsilon_{j} \epsilon_{1} e^{-i d\left(t_{j}-t_{1} / d-1 / d\right) \xi \overline{\widehat{a_{j, 1}}}(\xi)} .
\end{aligned}
$$

Again by (6), we know (22) is equivalent to

$$
\dot{\phi}_{j}(x)=\dot{\phi}_{j}\left(\dot{t}_{j}-x\right), \quad j=1, \ldots, r
$$

where $\dot{t}_{j}=t_{j}+\delta_{j-1}$ and $\dot{\phi}(x)=\left(\dot{\phi}_{1}(x), \ldots, \dot{\phi}_{r}(x)\right)^{T}$ is defined by $\widehat{\dot{\phi}}(d \xi)=\widehat{\dot{a}}(\xi) \widehat{\dot{\phi}}(\xi)$. In other words, compared with $\phi$, the components of $\dot{\phi}(x)$ are all symmetric.

From the aspect of two-scale similarity transforms (TST), $\hat{\dot{a}}(\xi)$ is the singular TST of $\widehat{a}(\xi)$. Therefore, by 
[14, Theorem 3.3.3], $\hat{\dot{a}}(\xi)$ has $m+1$ sum rules. Define a strongly invertible $r \times r$ matrix $\widehat{M}(\xi)$ as follows:

$$
\widehat{M}(\xi)=\left[\begin{array}{cccc}
0 & \cdots & 0 & 1 \\
0 & \cdots & 1 & \widehat{m}_{2 r}(\xi) \\
0 & \vdots & \vdots & \vdots \\
1 & \cdots & 0 & \widehat{m}_{r r}(\xi)
\end{array}\right],
$$

with $\widehat{m}_{j r}(\xi)$ satisfying $\widehat{m}_{j r}(\xi)=e^{-i d\left(\dot{t}_{1}-\dot{t}_{j} / d\right) \xi}{\widehat{\widehat{m}_{j r}}}_{(\xi)}$ such that

$$
\begin{aligned}
D^{\mu} & {\left.\left[\widehat{m}_{j r}(\xi) e^{i((j-1) / r) \xi} \widehat{\dot{\phi}}_{r}(\xi)+e^{i((j-1) / r) \xi} \widehat{\dot{\phi}_{r-j}}(\xi)\right]\right|_{\xi=0} } \\
& =\left.D^{\mu}\left[\widehat{\dot{\phi}}_{r}(\xi)\right]\right|_{\xi=0}, \quad \mu=0, \ldots, n-1, j=1, \ldots, r-1,
\end{aligned}
$$

where $t_{j}$ is as in (23).

$$
\begin{gathered}
\text { Construct } \phi_{\natural}(x)=\left(\phi_{\natural 1}(x), \ldots, \phi_{\natural r}(x)\right)^{T} \text { via } \\
\widehat{\phi_{\natural}}(\xi)=\widehat{M}(\xi) \widehat{\dot{\phi}}(\xi) .
\end{gathered}
$$

Then, by (7) and (8), we get

$$
\phi_{\natural j}(x)=\phi_{\natural j}\left(\dot{t}_{j}-x\right), \quad \dot{t}_{j} \in \mathbb{R}, j=1, \ldots, r .
$$

Moreover, (25) is equivalent to

$$
\begin{aligned}
\int_{\mathbb{R}} & \phi_{\natural 1}(x) x^{\mu} d x \\
& =\int_{\mathbb{R}} \phi_{\natural 2}(x)\left(x-\frac{1}{r}\right)^{\mu} d x \\
& =\cdots=\int_{\mathbb{R}} \phi_{\natural r}(x)\left(x-\frac{r-1}{r}\right)^{\mu} d x, \quad \mu=0, \ldots, n-1 .
\end{aligned}
$$

That is, $\phi_{\natural}$ has $n$ balanced orders. Select $\widehat{V}(\xi)$ := $\widehat{M}(\xi) \widehat{H}(\xi) \widehat{U}(\xi)$ and define $\widehat{a_{\natural}}(\xi)=(1 / d) \widehat{V}(d \xi) \widehat{a}(\xi) \widehat{V}(\xi)^{-1}$. Then, $\widehat{\phi_{\natural}}(d \xi)=\widehat{a_{\natural}}(\xi) \widehat{\phi_{\natural}}(\xi)$.

Next, we will prove that $\widetilde{\phi}_{\natural}$, which is defined via (18), is a dual refinable function vector of $\phi_{\natural}$. In fact,

$$
\begin{aligned}
& \widehat{a_{\natural}}(\xi) \widehat{\vec{a}_{\natural}}(\xi)^{*} \\
& =\frac{1}{d} \widehat{V}(d \xi) \widehat{a}(\xi) \widehat{V}(\xi)^{-1}\left(d \widehat{V}(d \xi)^{-*} \widehat{a}(\xi) \widehat{V}(\xi)^{*}\right)^{*} \\
& =\widehat{V}(d \xi) \widehat{a}(\xi) \widehat{a}(\xi)^{*} \widehat{V}(d \xi)^{-1}
\end{aligned}
$$

which together with

$$
\sum_{\ell=0}^{d-1} \widehat{a}\left(\xi+\frac{2 \pi \ell}{d}\right) \widehat{a}\left(\xi+\frac{2 \pi \ell}{d}\right)^{*}=I_{r}
$$

leads to the fact that $\sum_{\ell=0}^{d-1} \widehat{a_{\natural}}(\xi+2 \pi \ell / d) \widehat{\widetilde{a_{\natural}}}(\xi+2 \pi \ell / d)^{*}=I_{r}$. Thus, $\widetilde{\phi}_{\natural}=\left(\widetilde{\phi}_{\natural 1}(x), \ldots, \widetilde{\phi}_{\natural r}(x)\right)^{T}$ is a dual refinable function vector of $\phi_{\natural}$. By (7) and (8), we have

$$
\widehat{V}(\xi)=\widehat{\dot{S}}(\xi)^{-1} \overline{\widehat{V}(\xi)} \widehat{S}(\xi),
$$

from which we deduce that

$$
\begin{aligned}
\widehat{\widetilde{a}_{\natural}}(\xi) & =d \widehat{V}(d \xi)^{-*} \widehat{a}(\xi) \widehat{V}(\xi)^{*} \\
& =d \widehat{V}(d \xi)^{-*} \widehat{S}(d \xi)^{-1} \overline{\widehat{a}(\xi)} \widehat{S}(\xi) \widehat{V}(\xi)^{*} \\
& =\widehat{V}(d \xi)^{-*} \widehat{S}(d \xi)^{-1} \overline{\widehat{V}(d \xi)^{*}} \overline{\widehat{\vec{a}_{\natural}}(\xi)} \overline{\widehat{V}(\xi)^{-*}} \widehat{S}(\xi) \widehat{V}(\xi)^{*} \\
& =\hat{\dot{S}}(d \xi)^{-1} \overline{\widehat{\vec{a}_{\natural}}(\xi)} \widehat{\dot{S}}(\xi) .
\end{aligned}
$$

That is, $\tilde{\phi}_{\natural}$ is symmetric. Moreover, $\widetilde{\phi}_{\natural j}(x)=\widetilde{\phi}_{\natural j}\left(\dot{t}_{j}-x\right)$, $\dot{t}_{j} \in \mathbb{R}, j=1, \ldots, r$. From the aspect of two-scale similarity transforms (TST), $\widehat{\widetilde{a}}_{\natural}(\xi)$ is the singular inverse TST of $\widehat{a}(\xi)$. Therefore, by [14, Theorem 3.3.3], $\widehat{\widetilde{a}}_{\natural}(\xi)$ has $m-1$ sum rules.

Next, we summarize the process of constructing $\phi_{\natural}$ in Theorem 3.

Algorithm 5. Let $\phi$ be as in Theorem 3. Then, based on [2], $\widehat{V}(\xi)$ can be constructed through the following steps.

Step 1. By Theorem 3, construct a strongly invertible $r \times r$ matrix $\widehat{U}(\xi)$ of $2 \pi$-periodic trigonometric polynomials such that the mask symbol $\widehat{a^{u}}(\xi)=\widehat{U}(d \xi) \widehat{a}(\xi) \widehat{U}(\xi)^{-1}$ takes the property of (19). Moreover, $\widehat{a^{u}}(\xi)$ can generate a $d$-refinable function vector, whose symmetry takes the form of (14).

Step 2. Define $\widehat{H}(\xi)=\left[\begin{array}{cc}\left(1-e^{-i \xi}\right) & O \\ O & I_{r-1}\end{array}\right]$ and $\hat{\dot{a}}(\xi)=$ $(1 / d) \widehat{H}(d \xi) \widehat{a^{u}}(\xi) \widehat{H}(\xi)^{-1}$. Then, $\widehat{\dot{a}}(\xi)$ takes the form of $(21)$. Compared with $\phi$, all the components of the $d$-refinable function vector $\dot{\phi}(x)=\left(\dot{\phi}_{1}(x), \ldots, \dot{\phi}_{r}(x)\right)^{T}$, which is defined by $\widehat{\dot{\phi}}(d \xi)=\widehat{\dot{a}}(\xi) \widehat{\dot{\phi}}(\xi)$, satisfy (23).

Step 3. Define a strongly invertible $r \times r$ matrix $\widehat{M}(\xi)$ as in (24), with $\widehat{m}_{j r}(\xi)$ satisfying $\widehat{m}_{j r}(\xi)=e^{-i d\left(\dot{t}_{1}-\dot{t}_{j} / d\right) \xi}{\widehat{\widehat{m}_{j r}}}_{j r}(\xi)$ such that (25) holds.

Step 4. Construct $\phi_{\natural}$ via $\widehat{\phi_{\natural}}(\xi)=\widehat{M}(\xi) \widehat{\dot{\phi}^{s}}(\xi)$. Then, $\phi_{\natural}$ has $n$ balanced orders.

Step 5. Define $\widetilde{\phi}_{\natural}$ via (18). Then, it is the dual symmetric refinable function vector of $\phi_{\natural}$.

Proof of Theorem 4. If the mask symbol of $\phi=\left(\phi_{1}, \ldots, \phi_{r}\right)^{T}$ in Theorem 3 takes the form of (15), then the dual mask symbol of $\hat{\dot{a}}(\xi)$ is 


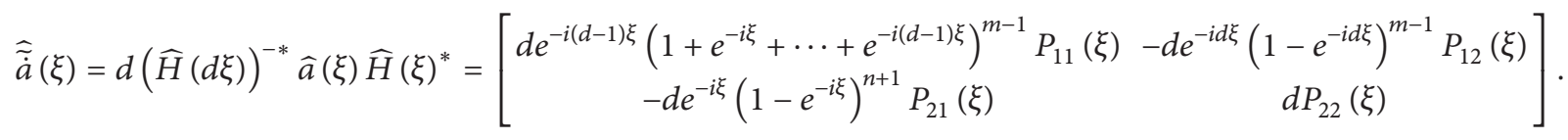

The transform matrix $\widehat{M}(\xi)$ in Step 3 of Algorithm 5 is strongly invertible. Therefore, the mask symbol $\widehat{\widetilde{a}}_{\natural}(\xi)=$ $\widehat{M}(d \xi)^{* *} \widehat{\tilde{\dot{a}}}(\xi) \widehat{M}(\xi)^{*}$ is a matrix of $2 \pi$-periodic trigonometric polynomials. That is, the dual refinable function vector $\widetilde{\phi_{\natural}}$, which satisfies $\widetilde{\phi_{\natural}}(d \xi)=\widetilde{\widehat{a}_{\natural}}(\xi) \widetilde{\phi_{\natural}}(\xi)$, is compactly supported.

\section{High Balanced Biorthogonal Multiwavelets from CL Multiwavelets}

In [12], Chui and Lian constructed an orthogonal refinable function vector $\phi(x)=\left(\phi_{1}(x), \phi_{2}(x)\right)^{T}$, which satisfies the following refinement equation:

$$
\widehat{\phi}(2 \xi)=\widehat{a}(\xi) \widehat{\phi}(\xi),
$$

where

$$
\widehat{a}(\xi)=\left[\begin{array}{ll}
\widehat{a_{11}}(\xi) & \widehat{a_{12}}(\xi) \\
\widehat{a_{21}}(\xi) & \widehat{a_{22}}(\xi)
\end{array}\right]
$$

with

$$
\begin{aligned}
\widehat{a_{11}}(\xi)= & \frac{10-3 \sqrt{10}}{80}\left(1+e^{-i \xi}\right) \\
& \times\left(1+(38+12 \sqrt{10}) e^{-i \xi}+e^{-i 2 \xi}\right), \\
\widehat{a_{12}}(\xi)= & \frac{5 \sqrt{6}-2 \sqrt{15}}{80}\left(1-e^{-2 i \xi}\right)\left(1+e^{-i \xi}\right), \\
\widehat{a_{21}}(\xi)= & \frac{5 \sqrt{6}-3 \sqrt{15}}{80}\left(1-e^{-i \xi}\right) \\
& \times\left(1-10(3+\sqrt{10}) e^{-i \xi}+e^{-i 2 \xi}\right), \\
\widehat{a_{22}}(\xi)= & \frac{5-3 \sqrt{10}}{1040}\left(1+e^{-i \xi}\right) \\
& \times\left(13-(10+6 \sqrt{10}) e^{-i \xi}+13 e^{-i 2 \xi}\right) .
\end{aligned}
$$

It is easy to see that $\widehat{a}(\xi)$ takes the form of (15) with $m=1$ and $n=1$. Specifically, straightforward observation gives us that $P_{11}(\xi)=((10-3 \sqrt{10}) / 80)\left(1+(38+12 \sqrt{10}) e^{-i \xi}+e^{-i 2 \xi}\right)$, $P_{12}(\xi)=((5 \sqrt{6}-2 \sqrt{15}) / 80)\left(1-e^{-2 i \xi}\right)$, and $P_{21}(\xi)=((5 \sqrt{6}-$ $3 \sqrt{15}) / 80)\left(1-10(3+\sqrt{10}) e^{-i \xi}+e^{-i 2 \xi}\right)$. Moreover, by [12], $\widehat{a}(\xi)$ has 3 sum rules. See Figure 1 for the graphs of $\phi_{1}(x)$ and $\phi_{2}(x)$. Select $\widehat{H}(\xi)=\left[\begin{array}{cc}\left(1-e^{-i \xi}\right) & 0 \\ 0 & 1\end{array}\right]$. Compute

$$
\begin{aligned}
& \widehat{\dot{a}}(\xi)=\frac{1}{2} \widehat{H}(2 \xi) \widehat{a}(\xi) \widehat{H}(\xi)^{-1}
\end{aligned}
$$

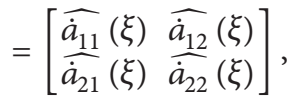

where

$$
\begin{aligned}
\widehat{\dot{a}_{11}}(\xi)= & \frac{10-3 \sqrt{10}}{160}\left(1+e^{-i \xi}\right)^{2} \\
& \times\left(1+(38+12 \sqrt{10}) e^{-i \xi}+e^{-i 2 \xi}\right), \\
\widehat{\dot{a}_{12}}(\xi)= & \frac{5 \sqrt{6}-2 \sqrt{15}}{160}\left(1-e^{-i \xi}\right)\left(1+e^{-i \xi}\right)^{2}\left(1-e^{-i 2 \xi}\right), \\
\widehat{\dot{a}_{21}}(\xi)= & \frac{5 \sqrt{6}-3 \sqrt{15}}{160}\left(1-10(3+\sqrt{10}) e^{-i \xi}+e^{-i 2 \xi}\right), \\
\widehat{\dot{a}_{22}}(\xi)= & \frac{5-3 \sqrt{10}}{2080}\left(1+e^{-i \xi}\right) \\
& \times\left(13-(10+6 \sqrt{10}) e^{-i \xi}+13 e^{-i 2 \xi}\right) .
\end{aligned}
$$

Then, by Theorem 3, the components of $\dot{\phi}(x)=$ $\left(\dot{\phi}_{1}(x), \dot{\phi}_{2}(x)\right)^{T}$, satisfying $\hat{\dot{\phi}}(2 \xi)=\hat{\dot{a}}(\xi) \hat{\dot{\phi}}(\xi)$, are all symmetric. $\hat{\dot{a}}(\xi)$ has 4 sum rules. By [3], we found out that the Sobolev exponent of $\dot{\phi}$ is 2.4408. According to Theorem 3, the dual refinable function vector $\widetilde{\dot{\phi}}(x)=\left(\widetilde{\dot{\phi}}_{1}(x), \widetilde{\dot{\phi}}_{2}(x)\right)^{T}$ of $\dot{\phi}(x)$ is given by $\widehat{\tilde{\dot{\phi}}}(2 \xi)=\widehat{\tilde{\dot{a}}}(\xi) \widehat{\tilde{\dot{\phi}}}(\xi)=2 \widehat{H}(2 \xi)^{-*} \widehat{a}(\xi) \widehat{H}(\xi)^{*} \widehat{\tilde{\dot{\phi}}}(\xi)$. That is,

$$
\begin{aligned}
\widehat{\dot{a}_{11}}(\xi)= & \frac{10-3 \sqrt{10}}{40} e^{-i \xi}\left(1+(38+12 \sqrt{10}) e^{-i \xi}+e^{-i 2 \xi}\right), \\
\widehat{\dot{a}_{12}}(\xi)= & -\frac{5 \sqrt{6}-2 \sqrt{15}}{40} e^{-i 2 \xi}\left(1+e^{-i \xi}\right), \\
\widehat{\dot{a}_{21}}(\xi)= & -\frac{5 \sqrt{6}-3 \sqrt{15}}{40} e^{-i \xi}\left(1-e^{-i \xi}\right)^{2} \\
& \times\left(1-10(3+\sqrt{10}) e^{-i \xi}+e^{-i 2 \xi}\right), \\
\widetilde{\dot{d}}_{22}(\xi)= & \frac{5-3 \sqrt{10}}{520}\left(1+e^{-i \xi}\right) \\
& \times\left(13-(10+6 \sqrt{10}) e^{-i \xi}+13 e^{-i 2 \xi}\right) .
\end{aligned}
$$

$\widehat{\tilde{\tilde{a}}}(\xi)$ has 2 sum rules. 


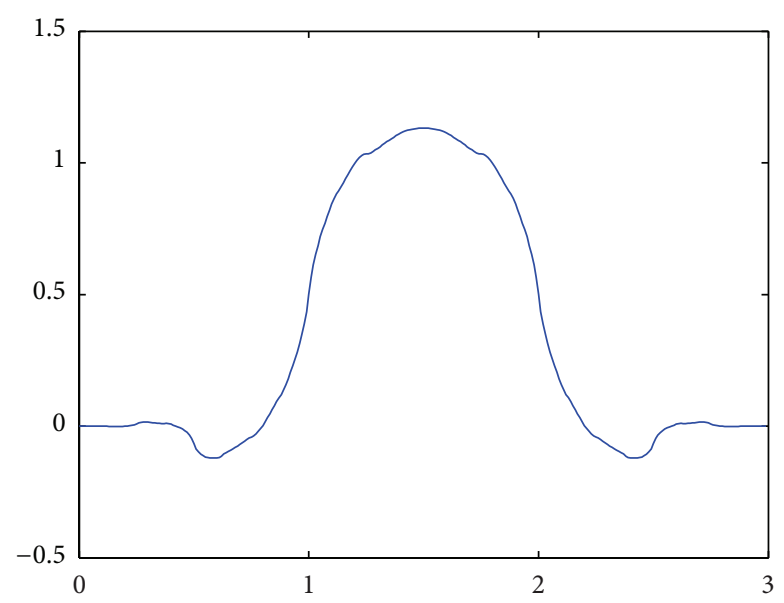

(a)

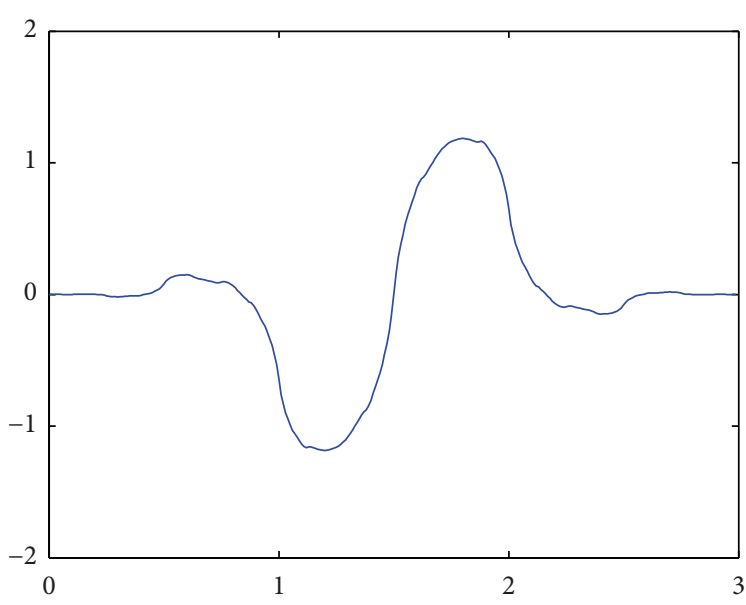

(b)

FIGURE 1: The graphs of $\phi_{1}(x)$ and $\phi_{2}(x)$ are shown on (a)-(b), respectively.

Next, we will construct balanced symmetric refinable function vectors and their dual refinable function vectors. We compute from $\widehat{\dot{\phi}}(2 \xi)=\hat{\dot{a}}(\xi) \widehat{\dot{\phi}}(\xi)$ that

$$
\begin{aligned}
\widehat{\dot{\phi}}(0) & =(-4.21498,1)^{T}, \\
D \widehat{\dot{\phi}}(0) & =(8.42996 i,-1.5 i)^{T}, \\
D^{2} \widehat{\dot{\phi}}(0) & =(17.44843,-2.24893)^{T} .
\end{aligned}
$$

We will find a $2 \pi$-periodic trigonometric polynomial and construct $\phi_{\natural}$ by

$$
\widehat{\phi_{\natural}}(2 \xi)=\widehat{M}(2 \xi) \hat{\dot{a}}(\xi) \widehat{M}(\xi)^{-1} \widehat{\phi_{\natural}}(\xi),
$$

where

$$
\widehat{M}(\xi)=\left[\begin{array}{cc}
0 & 1 \\
1 & \widehat{m}(\xi)
\end{array}\right],
$$

such that $\phi_{\natural}$ is balanced and symmetric.

By (25), if $\widehat{m}(\xi)$ satisfies $\widehat{m}(0)=5.21498$, then $\phi_{\natural}$ defined in (41) has 1 balanced order. Take $\widehat{m}(\xi)=5.21498\left(1+e^{-i \xi}\right) / 2$; for example, its graph is illustrated in Figures 2(a) and 2(b).

By (25), if

$$
\begin{aligned}
\widehat{m}(0) & =5.21498 \\
D \widehat{m}(0)= & -D \widehat{\dot{\phi}}_{1}(0)-\frac{i}{2} \widehat{\dot{\phi}}_{1}(0)-\frac{i}{2} \widehat{m}(0) \widehat{\dot{\phi}}_{2}(0) \\
& \left.\quad-(\widehat{m}(0)-1) D \widehat{\dot{\phi}}_{2}(0)\right) \times\left(\widehat{\dot{\phi}}_{2}(0)\right)^{-1} \\
= & -2.60749 i,
\end{aligned}
$$

then $\phi_{\natural}$ defined in (41) has 2 balanced orders. For this, construct

$$
\begin{aligned}
\widehat{m}(\xi)= & \left(1+e^{-i \xi}\right) \\
& \times\left[0.30374\left(e^{-i \xi}+e^{i \xi}\right)+\left(e^{-i 2 \xi}+e^{i 2 \xi}\right)\right]
\end{aligned}
$$

such that (43) is satisfied. Now, the graph of $\phi_{n}$, given by (41) with $\widehat{m}(\xi)$ being as in (44), is shown in Figures 2(c) and 2(d).

By (25), select $\widehat{m}(\xi)$, such that $\widehat{m}(\xi)=e^{-i \xi} \widehat{\bar{m}(\xi)}$ and

$$
\widehat{m}(0)=5.21498 \text {, }
$$

$$
\begin{aligned}
D \widehat{m}(0)= & -D \widehat{\dot{\phi}}_{1}(0)-\frac{i}{2} \widehat{\dot{\phi}}_{1}(0)-\frac{i}{2} \widehat{m}(0) \widehat{\dot{\phi}}_{2}(0) \\
& \left.\quad-(\widehat{m}(0)-1) D \widehat{\dot{\phi}}_{2}(0)\right) \times\left(\widehat{\dot{\phi}}_{2}(0)\right)^{-1} \\
= & -2.60749 i,
\end{aligned}
$$

$D^{2} \widehat{m}(0)$

$$
\begin{aligned}
=-\frac{1}{\widehat{\dot{\phi}}_{2}(0)}\left(\widehat{m}(0) D^{2} \widehat{\dot{\phi}}_{2}(0)+2\left(D \widehat{m}(0)+\frac{i \widehat{m}(0)}{2}\right)\right. \\
\quad \times D \widehat{\dot{\phi}}_{2}(0)+\left(\widehat{m}(0) \widehat{\dot{\phi}}_{2}(0)\left(\frac{i}{2}\right)^{2}+i D \widehat{m}(0)\right) \\
\quad \times \widehat{\dot{\phi}}_{2}(0)+D^{2} \widehat{\dot{\phi}}_{1}(0)-\frac{1}{4} \widehat{\dot{\phi}}_{1}(0) \\
\left.+i D \widehat{\dot{\phi}}_{1}(0)-D^{2} \widehat{\dot{\phi}}_{2}(0)\right)
\end{aligned}
$$$$
=-1.89673 .
$$

Construct $\widehat{m}(\xi)=\left(1+e^{-i \xi}\right)\left(\left(e^{-i \xi}+e^{i \xi}\right)+0.782280\left(e^{-i 2 \xi}+e^{i 2 \xi}\right)-\right.$ $\left.0.478534\left(e^{-i 3 \xi}+e^{i 3 \xi}\right)\right)$ such that (45) is satisfied. Then, $\phi_{b}$ has 3 balanced orders and is symmetric. See Figures 2(e) and 2(f) for its graph.

According to Algorithm 5, the mask symbol of $\widetilde{\phi}_{n}$, the dual refinable function vector of $\phi_{\natural}$, is $\widehat{\widetilde{a}}_{\natural}(\xi):=$ $\widehat{M}(2 \xi)^{-*} \widehat{\tilde{\dot{a}}}(\xi) \widehat{M}(\xi)^{*}$. By [3], we found that the Sobolev exponent of $\widetilde{\phi}_{\natural}$ is 0.4418 . 


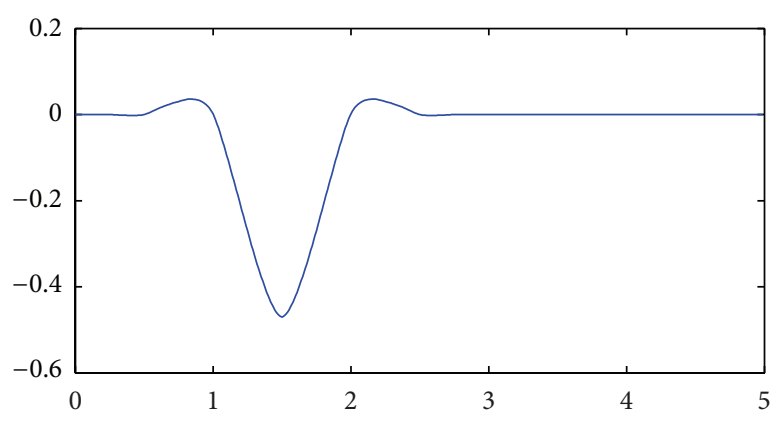

(a)

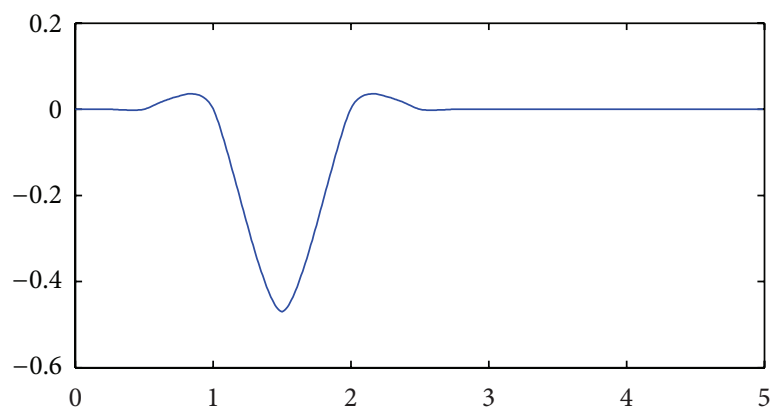

(c)

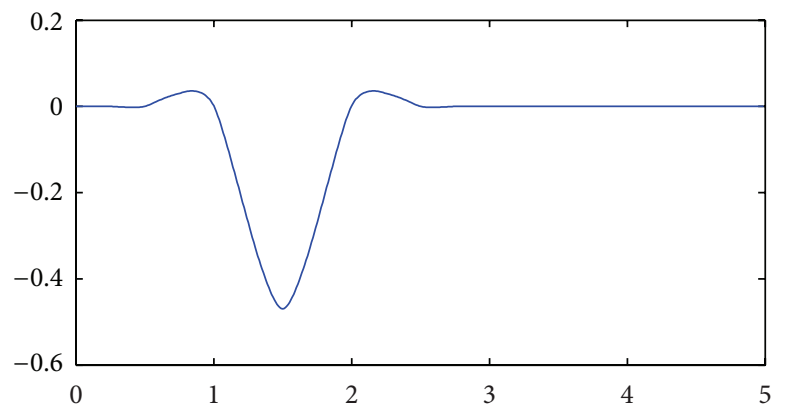

(e)

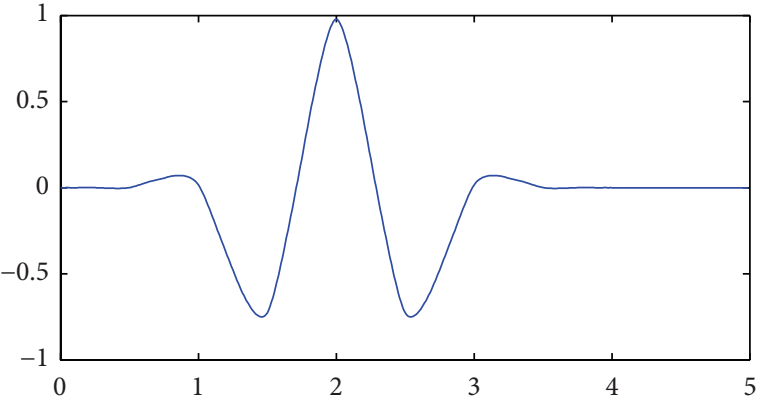

(b)

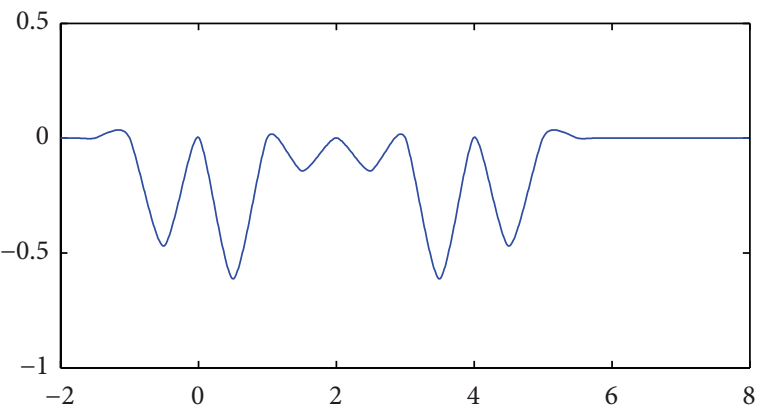

(d)

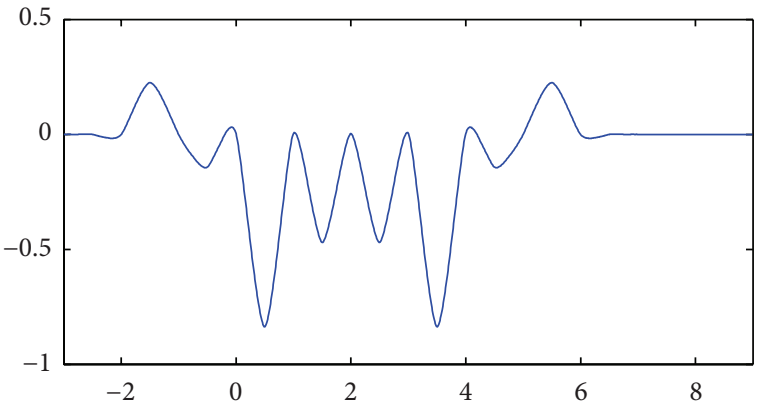

(f)

FIGURE 2: The graphs of $\phi_{\natural 1}(x)$ and $\phi_{\natural 2}(x)$ with 1 balanced order are shown on (a)-(b), respectively, while the ones with 2 balanced orders are shown on (c)-(d), and the ones with 3 balanced orders are shown on (e)-(f).

Moreover, for the pair of dual refinable function vectors, one can still get the dual multiwavelets. More specifically, suppose that $\widehat{b}(\xi)$ is the mask symbol of $\widehat{a}(\xi)$. Then, $\widehat{b_{\natural}}(\xi)$ and $\widetilde{\vec{b}_{\natural}}(\xi)$ defined by

$$
\begin{aligned}
& \widehat{\vec{b}_{\sharp}}(\xi)=2 \widehat{V}(2 \xi)^{-*} \widehat{b}(\xi) \widehat{V}(\xi)^{*}, \\
& \widehat{b_{\sharp}}(\xi)=\frac{1}{2} \widehat{V}(2 \xi) \widehat{b}(\xi) \widehat{V}(\xi)^{-1}
\end{aligned}
$$

are the mask symbols of their corresponding multiwavelets, where

$$
\widehat{V}(\xi)=\widehat{M}(\xi) \widehat{H}(\xi) .
$$

That is,

$$
\begin{aligned}
& \widehat{b_{\natural}}(\xi) \widehat{\widetilde{b}_{\natural}}(\xi)^{*}+\widehat{b}_{\natural}(\xi+\pi) \widehat{\widetilde{b}_{\natural}}(\xi+\pi)^{*}=I_{2}, \\
& \widehat{a_{\natural}}(\xi) \widehat{\widetilde{b}_{\natural}}(\xi)^{*}+\widehat{a_{\natural}}(\xi+\pi) \widehat{\widetilde{b}_{\natural}}(\xi+\pi)^{*}=O, \\
& \widehat{\widetilde{a}_{\natural}}(\xi) \widehat{b_{\natural}}(\xi)^{*}+\widehat{\widetilde{a}_{\natural}}(\xi+\pi) \widehat{b}_{\natural}(\xi+\pi)^{*}=O .
\end{aligned}
$$

\section{Future work}

In this paper, from an orthogonal refinable function vector $\phi$, which has antisymmetric component and is not balanced, we give an algorithm for constructing a pair of symmetric biorthogonal refinable function vectors $\phi_{n}$ and $\widetilde{\phi}_{\natural}$ such that $\phi_{\natural}$ has high balanced orders. When compared with $\phi$, the sum rule of $\phi_{\natural}$ increases by one while that of $\widetilde{\phi}_{\natural}$ decreases by 
one. Therefore, the Sobolev smoothness of $\widetilde{\phi}_{\natural}$ may be smaller than that of $\phi_{\natural}$. In future, based on $\widetilde{\phi}_{\natural}$, we will study how to construct a new symmetric and smooth dual refinable function vector of $\phi_{\natural}$.

\section{Conflict of Interests}

The authors declare that there is no conflict of interests regarding the publication of this paper.

\section{Acknowledgments}

The authors thank the two reviewers for their valuable suggestions which improve the paper a lot. The paper is supported by the Natural Science Foundation of Guangxi Province (no. 2013GXNSFBA019010), Natural Science Foundation of China (nos. 11126343, 11461002), and The Midwest Universities Comprehensive Strength Promotion Project of Guangxi University.

\section{References}

[1] Y. Huang and Y. Zhao, "Balanced multiwavelets on the interval $[0,1]$ with ar bitrary integer dilation factor $a$," International Journal of Wavelets, Multiresolution and Information Processing, vol. 10, no. 1, Article ID 1250010, 25 pages, 2012.

[2] B. Han and Q. Mo, "Multiwavelet frames from refinable function vectors," Advances in Computational Mathematics, vol. 18, no. 2-4, pp. 211-245, 2003.

[3] Q. Jiang, Matlab routines for Sobolev and holder smoothness comp utation of refinable functions, 2001, http://www.cs .umsl.edu/ jiang/Jsoftware.htm.

[4] B. Li, T. Luo, and L. Peng, "Balanced interpolatory multiwavelets with multiplicity $r$," International Journal of Wavelets, Multiresolution and Information Processing, vol. 10, no. 4, Article ID 1250039, 16 pages, 2012.

[5] R. Li and G. Wu, "The orthogonal interpolating balanced multiwavelet with rational coefficients," Chaos, Solitons and Fractals, vol. 41, no. 2, pp. 892-899, 2009.

[6] Y. Li, S. Yang, and D. Yuan, "Bessel multiwavelet sequences and dual multiframelets in Sobolev spaces," Advances in Computational Mathematics, vol. 38, no. 3, pp. 491-529, 2013.

[7] Y. Li, "Sampling approximation by framelets in Sobolev space and its application in modifying interpolating error," Journal of Approximation Theory, vol. 175, pp. 43-63, 2013.

[8] S. Yang and L. Peng, "Construction of high order balanced multiscaling functions via PTST," Science in China Series F: Information Sciences, vol. 49, no. 4, pp. 504-515, 2006.

[9] S. Yang and L. Peng, "Raising approximation order of refinable vector by increasing multiplicity," Science in China Series A, vol. 49, no. 1, pp. 86-97, 2006.

[10] Y. Shouzhi and W. Hongyong, "High-order balanced multiwavelets with dilation factor a," Applied Mathematics and Computation, vol. 181, no. 1, pp. 362-369, 2006.

[11] S. Yang, "Biorthogonal interpolatory multiscaling functions and corresponding multiwavelets," The ANZIAM Journal, vol. 49, no. 1, pp. 85-97, 2007.

[12] C. K. Chui and J.-A. Lian, "A study of orthonormal multiwavelets," Applied Numerical Mathematics, vol. 20, no. 3, pp. 273-298, 1996.
[13] F. Keinert, Wavelets and Multiwavelets, Studies in Advanced Mathematics, CRC Press, New York, NY, USA, 2003.

[14] V. Strela, Multiwavelets: Theory and Application, Department of Mathematics, Institute of Technology, Cambridge, Mass, USA, 1996. 


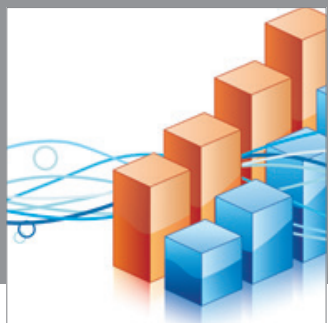

Advances in

Operations Research

mansans

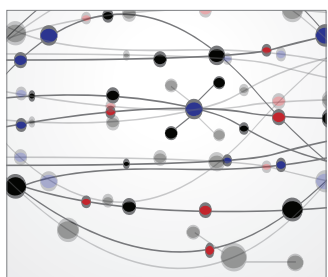

The Scientific World Journal
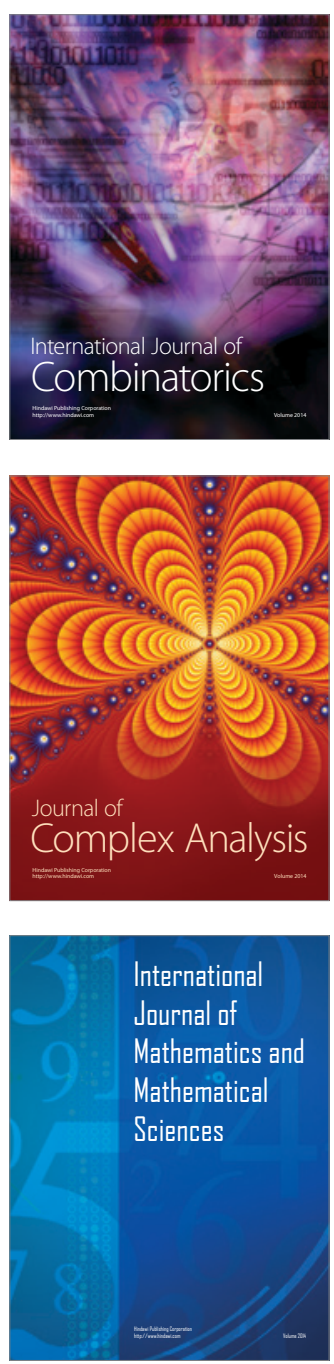
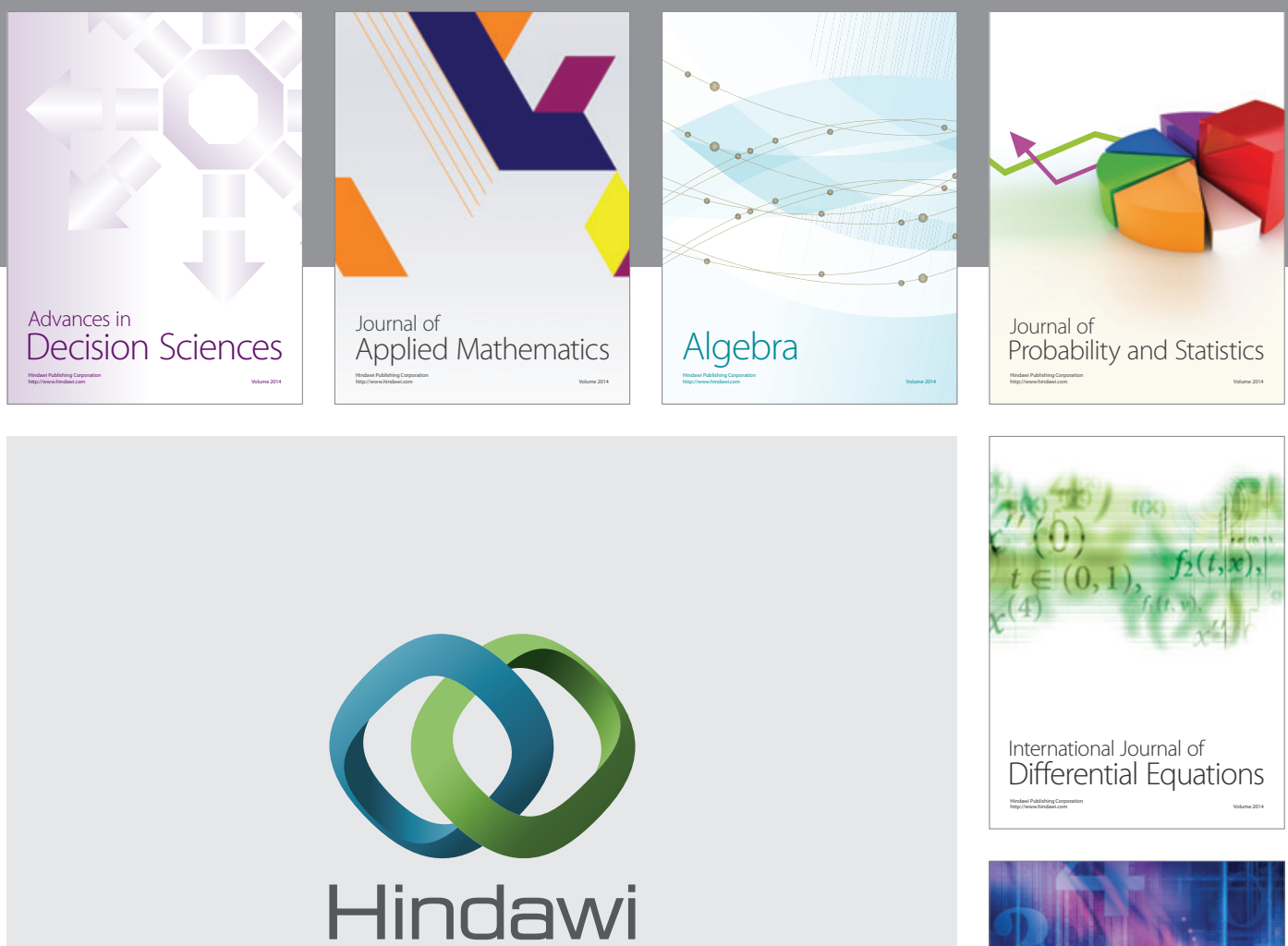

Submit your manuscripts at http://www.hindawi.com
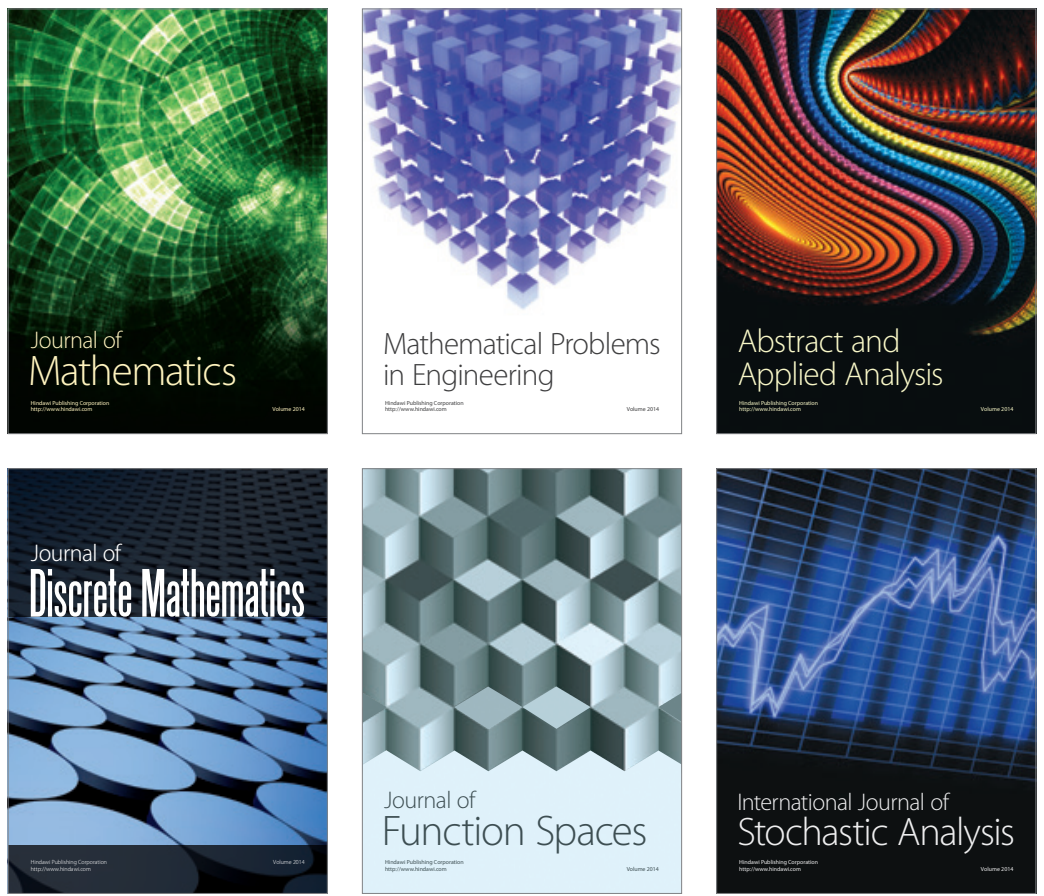

Journal of

Function Spaces

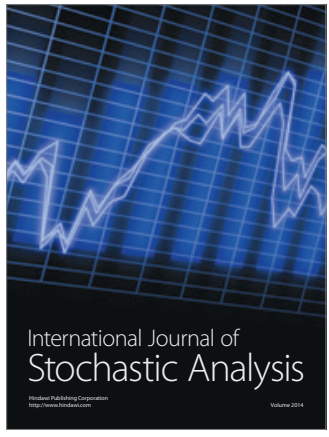

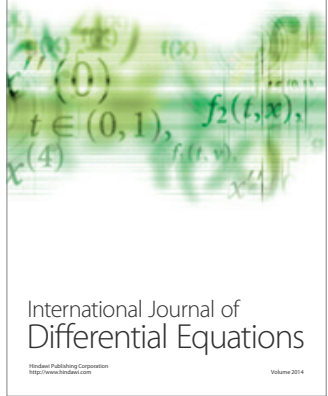
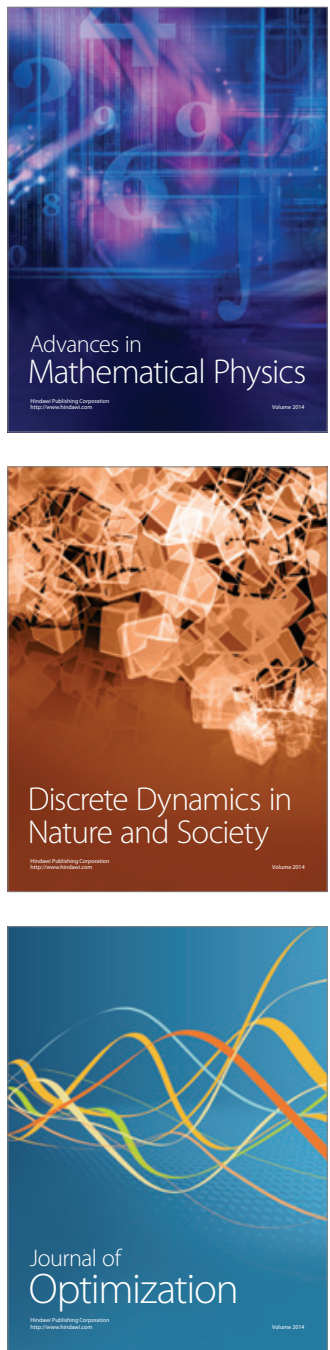